JGG 2021;69:188-194

doi: 10.36150/2499-6564-N241

Cuinical Geriatrics - Review

\title{
Dysphagia: what we know? A minireview
}

\author{
Tiziana Ciarambino ${ }^{1}$, Gennaro Sansone ${ }^{2}$, Ombretta Para ${ }^{3}$, \\ Mauro Giordano² \\ ${ }^{1}$ Internal Medicine Department, Hospital of Marcianise, ASL Caserta, Italy; ${ }^{2}$ Department of Medical, \\ Surgical, Neurological, Metabolic, and Geriatric Sciences, University of Campania L. Vanvitelli, Naples, \\ Italy; ${ }^{3}$ Department of Internal Medical, Hospital of Careggi, University of Florence, Italy
}

Dysphagia refers either to the difficulty someone may have with the initial phases of a swallow (usually described as oropharyngeal dysphagia, "OD") or to the sensation that foods and or liquids are somehow being obstructed in their passage from the mouth to the stomach (usually described as "esophageal dysphagia"). In patients with no indication of a somatic disease or abnormality, psychiatric conditions must be considered as a possible cause of OD. Moreover, diagnosis and treatment of dysphagia are not standardized. There is no universal standard tool for screening or clinical assessment of OD. Education of health professionals on early diagnosis and improvement of therapeutic strategies are mainstays to allow maximal recovery potential in this population. Future studies, clinical trials, clinical evidence and clear guidelines are needed to manage this condition.

Key words: dysphagia, oropharyngeal dysphagia, causes, guideline, management, elderly

\section{INTRODUCTION}

Accepted: April 20, 2021

\section{Correspondence \\ Tiziana Ciarambino \\ Internal Medicine Department, Hospital of Marcianise, ASL Caserta, via Orto dell'Abate, 81025, Marcianise (CE), Italy \\ E-mail: tiziana.ciarambino@gmail.com}

How to cite this article: Ciarambino $T$, Sansone G, Para O, et al. Dysphagia: what we know? A minireview. Journal of Gerontology and Geriatrics 2021;69:188-194. https://doi.org/10.36150/2499-6564-N241

(C) Copyright by Società Italiana

di Gerontologia e Geriatria (SIGG)

\section{(c) (1) () $\odot$}

\section{OPEN ACCESS}

This is an open access article distributed in accordance with the CC-BY-NC-ND (Creative Commons Attribution-NonCommercial-NoDerivatives 4.0 International) license. The article can be used by giving appropriate credit and mentioning the license, but only for non-commercial purposes and only in the original version. For further information: https://creativecommons.org/licenses/by-nc-nd/4.0/deed.en
Dysphagia refers either to the difficulty someone may have with the initial phases of a swallow (usually described as oropharyngeal dysphagia, "OD") or to the sensation that foods and or liquids are somehow being obstructed in their passage from the mouth to the stomach (usually described as "esophageal dysphagia") ${ }^{1}$. It is positive in $12.4 \%$ after extubation (18.3\% of emergency and $4.9 \%$ of elective patients) ${ }^{2}$. The prevalence of OD has been calculated in older people across different setting, with rates between 30 and $40 \%$ in geriatric acute care unit ${ }^{3,4}$. It may be more common in an acute hospital setting because patients often have multiple medical comorbidities. In particular, it observed predominantly in patients with an altered mental status ${ }^{5}$. Well-established risk factors for dysphagia include advanced age, neurological disease, head-neck cancer and pulmonary disease ${ }^{6}$. However, Giordano et al, have been reported that in an octogenarian, the only clinical symptoms of generalized tetanus, dysphagia and tremor ${ }^{7}$. It has been reported that isolated bulbar symptoms, as dysphagia, in elderly, may be included in the differential diagnosis ${ }^{8}$. To this regard, the European Society for swallowing disorders-European Union Geriatric Medicine Society describe that dysphagia can cause severe complications such as malnutrition, dehydration, respiratory infections, aspiration/pneumonia and increased readmissions, institutionalization and mortality. In fact, $\mathrm{OD}$ is a prevalent and serious problem in older patients. Oro-pharyngeal swallow response is impaired in older people and can 
cause aspiration ${ }^{9}$. Despite its prevalence and severity, $\mathrm{OD}$ is still underdiagnosed and untreated in many medical centers.

The present minireview highlights the importance of considering crucial causes among the possible expression and cause of dysphagia.

\section{METHODS}

Clinical trials were identified by PubMed until June 30 2020. The search keywords were "dysphagia, elderly, OD". The studies were selected, their references were reviewed for potential inclusion. Studies written in languages other than English were excluded. Three authors (T.C., G.S., and O.P.) reviewed all study abstracts. Studies were included if analyzed dysphagia in elderly patients All selected studies are qualitatively analyzed.

\section{ETIOPATHOGENESIS}

Dysphagia can be related to gastrointestinal or not gastrointestinal causes, as reported in Table I.

\section{GASTROINTESTINAL CAUSES}

\section{Eosinophilic esophagitis}

Esophageal food bolus impaction is an acute multidisciplinary common emergency, frequently observed in the Emergency Department ${ }^{10}$. The estimated annual incidence rate is 13 per 100,000 persons after upper and lower gastrointestinal bleeding ${ }^{11}$. The most common presentation of food bolus impaction in the Western countries are the "steakhouse syndrome", so called because the meats represent the most frequently impacted foods ${ }^{12}$. Several abnormalities of esophageal mucosal and neuromuscular layers, as well as underlying diseases, including edentulism, eosinophilic esophagitis and esophageal stenosis (as peptic esophageal

Table I. Gastrointestinal and not gastrointestinal causes of dysphagia.

\begin{tabular}{|l|c|}
\hline Causes & Prevalence (\%) \\
\hline Gastrointestinal & Eosinophilic esophagitis (30-54\%) \\
& Reflux esophagitis (36\%) \\
& Barrett' (10\%) \\
& Other (30\%) \\
Not gastrointestinal & Post-stroke dysphagia (2\%) \\
\hline & Dementia (19-30\%) \\
& M. Parkinson (35\%) \\
& M. Alzheimer (57-84\%) \\
\hline
\end{tabular}

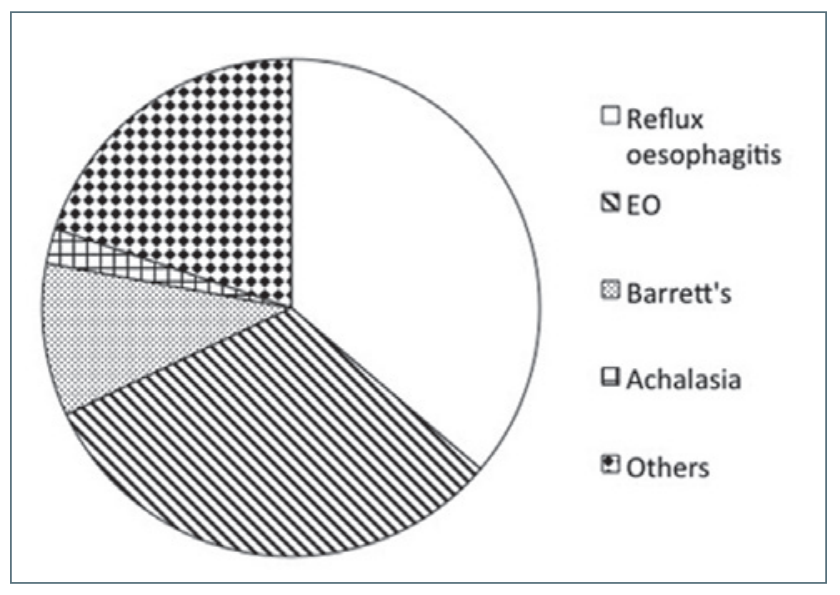

Figure 1. In this figure we describe the main gastrointestinal causes of dysphagia. Eosinophilic esophagitis (E0).

stricture or Schatzki's ring), facilitate food bolus impaction ${ }^{13}$. In particular, several studies suggest that eosinophilic esophagitis (EO) is an emergency cause of food bolus impaction and dysphagia in adult (Fig. 1) ${ }^{14,15}$. It has been reported that patients who present with food bolus impaction, have approximately 1 in 3 chance of having EO. According to a study, EO was found in 54\% of patients presenting with food bolus impaction ${ }^{16}$. EO usually affects young adults with mean age 30 years old ${ }^{14}$. The exclusion of secondary causes of EO, the presence of mucosal eosinophilia being isolated to the esophagus and persisting after a proton pump inhibitor trial are also included in the diagnostic criteria for EO ${ }^{17}$. According to the current guidelines, EO is defined histologically with eosinophils count of 15 or more eosinophils/hpf.

\section{Esophageal motility disorders}

More infrequently, esophageal motility disorders (as diffuse esophageal spasm) and other esophageal hypertensive dysmotility's, may represent a cause of food bolus impaction ${ }^{18}$. This multidisciplinary, as well as the plurality of underlying esophageal diseases, generate a high heterogeneity in its management, ranging from observational therapy to surgical treatment ${ }^{19}$. Although more than half of food bolus impaction will resolve spontaneously after a short observational period without any serious consequences, in $10-20 \%$ of cases a conservative or surgical management is required ${ }^{20}$. Several studies have been conducted on pharmacological and non-pharmacological agents for treatment of this issue. Pharmacological agents with enzymatic activity such as papain, trypsin and chymotrypsin are no longer recommended due to high risk of esophageal perforation and hypernatremia. In the same way, the administration of hyoscine butyl-bromide (Buscopan), 
glucagon injection and diazepam resulted in no significant difference in compare to controls ${ }^{21}$. Better results were obtained by non-pharmacological effervescent agents (fizzy drinks). However, the level of evidence of these studies is too low to provide strong conclusions. The current treatment, recommended by American Society for Gastrointestinal Endoscopy (ASGE) include endoscopic food extraction and, only in some cases, the advancement of the bolus into the stomach under endoscopic guide ${ }^{22}$. With regards to the management of food bolus impaction, Ikenberry et al., recommended a time frame of $6 \mathrm{~h}$ to remove the food bolus due to the potential risk of ischemia, necrosis and perforation ${ }^{23}$.

\section{Achalasia}

It is a relatively rare primary motor esophageal disorder, characterized by absence of relaxation of the lower esophageal sphincter and of peristalsis along the esophageal body. As a result, patients typically, present with dysphagia, regurgitation and occasionally chest pain. High resolution manometry is recommended for the diagnosis of the esophageal achalasia (GRADE recommendation: (ow). It is recommended to perform endoscopy to exclude neoplastic pseudo-achalasia (Good practice recommendation). There is no convincing evidence that medical treatment with nitrates or calcium blocker or phosphodiesterase inhibitors is effective for symptomatic relief in adult with achalasia. Treatment of achalasia with peroral endoscopic myotomy (POEM) results in similar outcomes on swallowing functions compared with alternative treatment ${ }^{24}$.

\section{NON-GASTROINTESTINAL CAUSES}

\section{Post-stroke dysphagia}

Dysphagia is a common complication after stroke with a prevalence to vary from 51 to $65 \%$ of patients. Many of them are asymptomatic or have symptoms that aren't related to swallowing problems. Although many patients recover swallowing spontaneously, 11-50\% still have dysphagia at 6 months ${ }^{25,26}$. Post-stroke dysphagia (PSD) is associated with a poor outcome for multiple reasons:

- it is a manifestation of severe stroke and it is associated with increased death, dependency, disability, impairment and institutionalization;

- it causes aspiration of foods, liquids and oral secretions and therefore pneumonia ${ }^{27,28}$, which in itself leads to death;

- however, poor recognition and management leads to dehydration and malnutrition ${ }^{29}$.

Although multiple advances have been made in the early management of stroke (e.g. with thrombolysis, aspirin, mechanical thrombectomy and hemicraniectomy) and secondary prevention (e.g. with antithrombotic, blood pressure lowering, lipid lowering, carotid endarterectomy), PSD remains a neglected research area and its optimal management, including treatment, has yet to be defined. Nevertheless, guidelines recommend assessment of swallowing within $24 \mathrm{~h}$ after stroke ${ }^{1}$. In respect of drug treatment, a small pilot randomized trial suggested that nifedipine (a calcium channel blocker that relaxes esophageal smooth muscle) might improve swallowing, and metoclopramide (a dopamine D2-receptor antagonist with antiemetic and gastric prokinetic activity) might reduce the incidence of pneumonia ${ }^{25}$. Glyceryl trinitrate (GTN), given within 6h of stroke, was associated with improved functional outcome ${ }^{30,31}$. In fact, GTN might also improve swallowing though relaxing esophageal smooth muscle ${ }^{32-34}$.

\section{Medically unexplained oropharyngeal dysphagia (MUNOD)}

Rarely, OD occurs without demonstrable abnormalities in the anatomy of the upper aero-digestive tract and/or swallowing physiology, prompting a diagnosis of medically unexplained oropharyngeal dysphagia (MUNOD). Functional somatic disorders and comorbid anxiety and depression are both associated with increased severity of symptoms and greater illness burden. According to the DSM-V classification, phagophobia belongs to the category of 'specific phobias' (26). Patients with phagophobia experience an abnormal sensation during swallowing, sometimes accompanied by behavioral abnormalities during swallowing examination ${ }^{35}$.

\section{MANAGEMENT}

Thus, patients with a food bolus impaction in the emergency department should receive a clinical and instrumental assessment with chest radiograph to rule out evidence of perforation or a radiopaque object in the esophagus (Fig. 2). Once a foreign object is ruled out, endoscopy should be considered ${ }^{36}$. The food bolus can be removed during upper endoscopy and is successful in $98 \%$ of patients during the first endoscopy ${ }^{37}$. A recent review explored the level of evidence and classified the types of interventions into the following categories:

- bolus modification and management;

- swallow postures and maneuvers;

- other interventions (as oral hygiene, feeding);

- facilitation techniques.

\section{BOLUS MODIFICATIONS}

The modification of the consistence of solids and or liquids is the main element of compensatory treatment for 


\section{Screning all patients at risk of dysphagia}
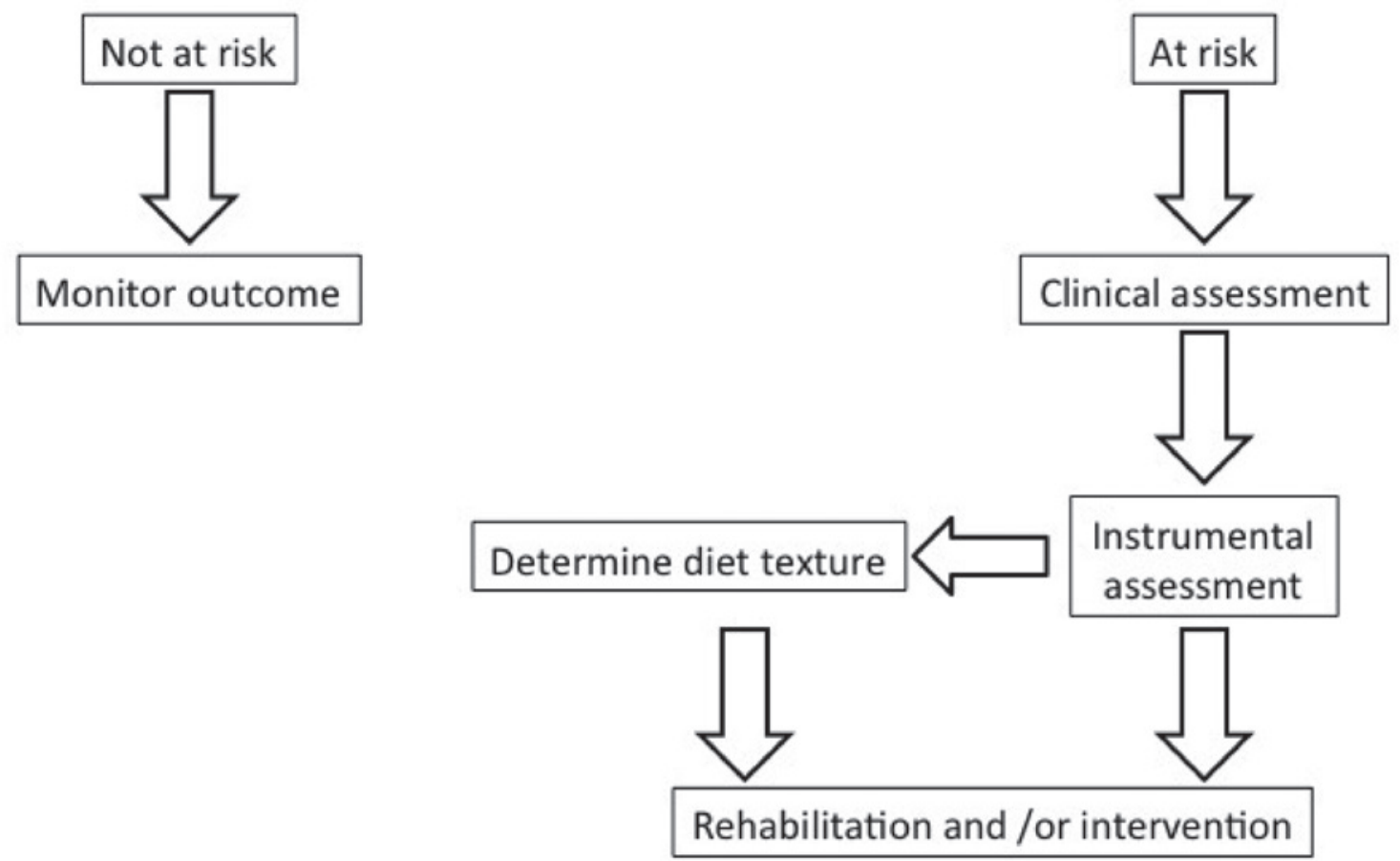

Figure 2. In this figure we describe the algorithm to use for the management of the dysphagia.

patients suffering from OD. The level of evidence with this treatment is A (randomized controlled trials) and B (non-randomized trials) ${ }^{38}$.

\section{SWALLOW POSTURE}

A general directive is to swallow in an upright position (90 seated) and to maintain this posture after the meal for at least 30 minutes. Generally, level of evidence for maneuvers or other posture is $\mathrm{B}^{38}$.

\section{ORAL HYGIENE}

Deficient oral hygiene is a risk factor for pneumonia in older patients with $\mathrm{OD}^{39}$. Minimal oral hygiene should be performed every 12 hours to avoid dental plaque formation ${ }^{40}$. Mouthwashes should be used at least every 3 days, chlorhexidine being the most effective but it should not be used for $>15$ days ${ }^{41}$.

\section{NUTRITIONAL STATUS}

Nutritional status is basic to good health and an important part of treatment of many chronic diseases. The relationship between malnutrition and OD has been established. It has been recommended that patients following modified texture diet or being given enteral feeding for OD should have their swallowing and nutritional status regularly assessed, after the first week and then every 2 or 3 months for the first years and after every 6 months ${ }^{42}$.

Oral nutritional supplement should be provided in patients with:

- anorexia;

- dietary restriction due to chronic disease;

- nutritional intake $<75 \%$ of their nutritional requirements;

- involuntary weight loss.

Nutritional interventions include different strategies such as adaptation of the diet and enteral nutrition, administered by nasogastric tube (NGT) or percutaneous endoscopic gastrostomy (PEG) and should be based on the nutritional needs.

NGT is recommended for short periods of time $(<2$ months) and when there is no risk of gastro esophageal reflux. It is reported for the care of the patients with amyotrophic lateral sclerosis ${ }^{43}$.

PEG is most commonly used and it can be inserted by endoscopy or surgery. Carrau et al., reported the use of laryngeal framework surgery for the effective treatment of aspiration in selected patients (such as with deficits of the glottic closure secondary to vocal fold paralysis or paresis) ${ }^{44}$. PEG may cause an enhanced risk of gastro 
esophageal reflux disease (GERD) with increased risk of aspiration ${ }^{45}$. The recommendations by Wirth et al., underline the importance of an early screening and assessment of dysphagia and give advice for an evidence based and comprehensive nutritional management to avoid aspiration, malnutrition and dehydration ${ }^{46}$.

\section{FACILITATION TECHNIQUES}

In recent years, new treatment based on stimulation or sensorial and motor pathways are being assessed. Neuromuscular electrical stimulation therapy (NMES) stimulates deglution nerves and muscles to improve $\mathrm{OD}$. The main target nerves are the superior laryngeal nerve, the pharyngeal branch of the glossopharyngeal nerve and two branches of the vagus nerve, the pharyngeal branch and the maxillary branch of the trigeminal nerve. Guidelines on the NMES have been published by the British National Institute for Health and Care Excellence giving recommendation. The evidence on safety is limited in quality and quantity. In patients with PSD, treatment with transcutaneous NMES increased prevalence of safe swallows and reduced the time of the laryngeal vestibule closure (LVC) ${ }^{47}$.

\section{OTHER EVALUATION}

In addition, MUNOD is a rare condition that is difficult to diagnose. Patients deserve a professional approach, particularly because their diagnostic trajectory has often been long and inconclusive. Affective symptoms are common in these patients. MUNOD could be a symptom of a psychiatric condition or part of the alarm falsification defense system, suggesting that physical symptoms and affective disorders are stress-related and a response to earlier threats. Consultation of a psychiatrist for patients with MUNOD is recommended as part of a pathway toward multidisciplinary integrated care ${ }^{35}$. In patients with prolonged dysphagia, with no indication of a somatic disease or abnormality, psychiatric conditions must be considered as a possible cause of OD. Validated psychological screening questionnaires could be helpful in the detection of affective conditions but also of other psychiatric conditions. Involvement of a psychiatrist and/or psychologist is recommended.

\section{DISCUSSION}

The present review describes the gastrointestinal and not gastrointestinal causes of dysphagia.

Due to its prognostic importance, an early detection of causes related dysphagia and a suitable nutritional management is therefore of utmost clinical importance ${ }^{46}$. A formalized screening for dysphagia should be carried out in all patients as part of the initial examination or upon arrival of the patient on the hospital ward. To this regard, several studies reported associations between a pathological dysphagia screening and an increased incidence of pneumonia ${ }^{48}$ as well as a reduction of infectious complications after implementation of a systematic screening ${ }^{49}$. Unfortunately, despite its prevalence and severity, dysphagia is still underdiagnosed and undertreated in many medical centers. Moreover, diagnosis and treatment of dysphagia are not standardized. There is no universal standard tool for screening or clinical assessment of OD. Education of health professionals on early diagnosis and improvement of therapeutic strategies are mainstays to allow maximal recovery potential in this population ${ }^{50}$.

Current treatment of $\mathrm{OD}$ is usually compensatory, rehabilitative or a combination of the two. Future studies, clinical trials, clinical evidence and clear guidelines are needed to manage this condition.

\section{Ethical consideration}

None.

\section{Acknowledgement \\ None.}

\section{Funding}

This research received no external funding.

\section{Conflict of interest}

The Authors declare no conflict of interest.

\section{References}

1 Malagelada JR, Bazzoli F, Boeckxstaens G. World gastroenterology organization global guidelines: dysphagia global guidelines and cascades update September 2014. J Clin Gastroenterol 2015;49:370. https://doi.org/10.1097/ MCG.0000000000000307

2 Schefold J, Berger D, Zurcher P, et al. Dysphagia in mechanically ventilation ICU patients (Dynamics): a prospective observational trial. Crit Care 2017;45:2061-2069. https://doi.org/10.1097/CCM.0000000000002765

3 Cabre M, Almirall J, Clave P. Aspiration pneumonia: management in Spain. Eur Geriatr Med 2012;3:180-183.

4 Clave P, Rofes L, Carrion S. Pathophysiology, relevance and natural history of OD among older people. Nestle Nutr Inst Workshops Ser 2012;72:57-66. https://doi. org/10.1159/000339986

5 Simning I, Simning A. Severe idiopathic dysphagia in an acute hospital setting: assessment, management, and outcome. J Med Speech Lang Pathol 2013;21:3.

6 Garon B, Sierzant T, Ormiston C. Silent Aspiration: results of 2,000 video fluoroscopic evaluations. J Neurosci Nurs 2009;41:178-185. 
7 Giordano M, Ciarambino T, Di Bella O. Acute dysphagia in an octogenarian: an unusual case of tetanus. Am J Emerg Med 2014;32:691.e1-691.e2. https://doi.org/10.1016/j. ajem.2013.12.020

8 Mustafa M, Mohamed MB, Hayat A. Tumour lysis syndrome and partial remission occurring after administration of a test dose of obinutuzumab. Eur J Case Rep Intern Med 2017;4:000516. https://doi.org/10.12890/2016_000516

9 Baijens LWJ, Clave P, Cras O. European Society for swallowing disorders-European Union Geriatric Medicine Society white paper: oropharyngeal dysphagia as a geriatric syndrome. Clin Interv Aging 2016,11:1403-1428.

10 Price $\mathrm{T}$, Jones SE, Montgomery PQ. Is current UK management of oesophageal food bolus obstruction evidence based? An e-mail survey and literature review. Eur Arch Otorhinolaryngol 2007;264:329-335. https://doi. org/10.1007/s00405-007-0260-x

11 Longstreth GF, Longstreth KJ, Yao JF. Esophageal food impaction: epidemiology and therapy. A retrospective, observational study. Gastrointest Endosc 2001;53:193-198. https://doi.org/10.1067/mge.2001.112709

12 Stadler J, Holscher AH, Feussner H, et al. The "Steakhouse syndrome" - primary and definitive diagnosis and therapy. Surg Endosc 1989;3:195-198. https://doi.org/10.1007/ BF02171545

13 Sperry SL, Crockett SD, Miller CB, et al. Esophageal foreign-body impactions: epidemiology, time trends, and the impact of the increasing prevalence of eosinophilic esophagitis. Gastrointest Endosc 2011;74:985-991. https:// doi.org/10.1016/j.gie.2011.06.029

${ }^{14}$ Heerasing N, Lee SY, Alezander S. Prevalence of eosinophilic oesophagitis in adults presenting with oesophageal food bolus impaction. WJG Pharmacol Therap 2015;6:244-247. https://doi.org/10.4292/wjgpt.v6.i4.244

15 Attwod SE, Forutaa GT. Eosinophilic esophagitis: historically perspective on an evolving disease. Gastroenterol Clin North Am 2014;43:185-199. https://doi.org/10.1016/j. gtc.2014.02.010

16 Desai TK, Stecevic V, Chang $\mathrm{CH}$. Association of eosinophil inflammation with esophageal food impaction in adult. Gastrointest Endosc 2005,61:795-801. https://doi. org/10.1016/s0016-5107(05)00313-5

17 Furuta GT, Liacouras CA, Collins MH et al. Eosinophilic esophagitis in children and adults: a systematic review and consensus recommendations for diagnosis and treatment. Gastroenterology 2007;133:1342-1363. https://doi. org/10.1053/j.gastro.2007.08.017

18 Chae HS, Lee TK, Kim YW, et al. Two cases of steakhouse syndrome associated with nutcracker esophagus. Dis Esophagus 2002;15:330-333. https://doi. org/10.1046/j.1442-2050.2002.00271.x

19 Reddy VM, Bennett W, Burrows SA, et al. Recurrence of food bolus impaction of the oesophagus: a retrospective observational study. Int J Surg 2011;9:464-466. https:// doi.org/10.1016/j.ijsu.2011.04.011
20 Basavaraj S, Penumetcha KR, Cable HR, et al. Buscopan in oesophageal food bolus: is it really effective? Eur Arch Otorhinolaryngol 2005;262:524-527. https://doi. org/10.1007/s00405-004-0852-7

21 Khayyat YM. Pharmacological management of esophageal food bolus impaction. Emerg Med Int 2013;2013:924015. https://doi.org/10.1155/2013/924015

22 ASGE Standards of Practice Committee (https://www. asge.org/home/resources/key-resources/guidelines)

23 Ikenberry SO, Jue TL, Anderson MA. Management of ingested foreign bodies and food impactions. Gastrointes Endosc 2011;73:1085-1091. https://doi.org/10.1016/j. gie.2010.11.010

24 Zaninotto G, Bennett C, Boeckxstaens G. The 2018 ISDE achalasia guidelines. Dis Esophagus 2018;31:1-29. https://doi.org/10.1093/dote/doy071

25 Martino R, Foley N, Bhogal S, et al. Dysphagia after stroke: incidence, diagnosis, and pulmonary complications. Stroke 2005;36:2756-2763. https://doi.org/10.1161/01. STR.0000190056.76543.eb

26 Mann G, Hankey GJ, Cameron D. Swallowing disorders following acute stroke: prevalence and diagnostic accuracy. Cerebrovasc Dis 2000;10:380-386.

27 Smithard D, Smeeton N, Wolfe C. Long-term outcome after stroke: does dysphagia matter? Age Ageing 2007;36:9094. https://doi.org/10.1159/000016094

28 Rofes L, Arreola V, Almirall J, et al. Diagnosis and management of oropharyngeal dysphagia and its nutritional and respiratory complications in the elderly. Gastroenterol Res Pract 2011;2011:13. https://doi. org/10.1155/2011/818979

29 Wilson R. Mortality and cost of pneumonia after stroke for different risk groups. J Stroke Cerebrovasc Dis 2012;21. https://doi.org/10.1016/j. jstrokecerebrovasdis.2010.05.002

30 Warusevitane A, Karuntilake D, Lally F, et al. Safety and effect of metoclopramide to prevent pneumonia in patients with stroke fed via nasogastric tubes trial. Stroke 2015;46:454-460. https://doi.org/10.1161/ STROKEAHA.114.006639

31 Woodhouse L, Scutt P, Krishnan K, et al. Effect of hyperacute administration (within 6 hours) of transdermal glyceryl trinitrate, a nitric oxide donor, on outcome after stroke: subgroup analysis of the efficacy of nitric oxide in stroke (ENOS) trial. Stroke 2015;46:3194-3201.

32 Ankolekar S, Fuller M, Cross I, et al. Feasibility of an ambulance- based stroke trial, and safety of glyceryl trinitrate in ultra-acute stroke: the rapid intervention with glyceryl trinitrate in Hypertensive Stroke Trial (RIGHT, ISRCTN66434824). Stroke 2013;44:3120-3128. https://doi. org/10.1161/STROKEAHA.113.001301

${ }_{33}$ Wei J, Wu T, Yang Q, et al. Nitrates for stable angina: a systematic review and meta-analysis of randomized clinical trials. Int J Cardiol 2011;146:4-12. https://doi.org/10.1016/j. ijcard.2010.05.019 
34 Perez I, Smithard DG, Davies H, et al. Pharmacological treatment of dysphagia in stroke. Dysphagia 1998;13:1216. https://doi.org/10.1007/PL00009543

35 Baijens LW, Koetsenruijter K, Pilz W. Diagnosis and treatment of phagophobia: a review. Dysphagia 2013;28:260270. https://doi.org/10.1007/s00455-013-9454-0

36 VerdonschoT JCG, Laura WJ. Medically Unexplained oropharyngeal dysphagia at the University Hospital ENT outpatient clinic for dysphagia: a cross-sectional cohort study. Dysphagia 2019;34:43-51.

37 Vicari JJ, Johanson JF, Frakes JT. Outcomes of acute esophageal food impaction: success of the push technique. Gastrointest Endosc 2001;53:178-181. https://doi. org/10.1067/mge.2001.111039

38 Speyer R, Baijens L, Heijnen M. Effects of therapy in OD by speech and language therapist: a systematic review. Dysphagia 2010;25:40-65. https://doi.org/10.1007/ s00455-009-9239-7

39 Ortega O, Clave P. Oral hygiene, aspiration, and aspiration pneumonia from pathophysiology to therapeutic strategies. Curr Phys Med Rehabil Rep 2013:292-295.

40 Sjogren P, Nilsson E, Forseel M. A systematic review of the preventive effect of oral hygiene on pneumonia and respiratory tract infection in elderly in hospitals and nursing homes. JAGS 2008;56:2114-2130. https://doi. org/10.1111/j.1532-5415.2008.01926.x

41 Berchier CE, Slot DE, Van der Wenden GA. The efficacy of $0.12 \%$ chlorhexidine mouthrise compared with $0.2 \%$ on plaque accumulation and periodontal parameters: a systematic review. J Clin Periodont 2010;37:829-839.

42 European Society for swallowing disorders: position statement. Dysphagia 2013:28:280-335.
43 Miller RG, Rosenberg JA, Gelinas NF. Practice parameter: the care of the patient with amyotrophic lateral sclerosis (an evidence-based review): report of the Quality Standards Subcommittee of the American Academy of Neurology: ALS Practice Parameters Task Force. Neurology 1999;52:1311-1323.

44 Carrau RL, Pou A, Eibling DE, et al. Laryngeal framework surgery for the management of aspiration. Head Neck 1999;21:139145. https://doi.org/10.1002/(sici)1097-0347(199903)21:2

45 Groher ME. Determination of the risks and benefits of oral feeding. Dysphagia 1994;4:233-235. https://doi. org/10.1007/BF00301916

46 Wirth R, Smoliner C, Jäger M, et al.; The DGEM Steering Committee. Guideline clinical nutrition in patients with stroke. Experimental \& Translational Stroke Medicine 2013;5:14.

47 Gallas S, Marie JP, Leroi AM. Sensory transcutaneous electrical stimulation improve post stroke dysphagia. Dysphagia 2010;24:291-297. https://doi.org/10.1007/ s00455-009-9259-3

48 Sellars C, Bowie L, Bagg J, et al. Risk factors for chest infection in acute stroke. A prospective cohort study. Stroke 2007:38:2284-2291.

49 Lakshminarayan K, Tsai AW, Tong $\mathrm{X}$, et al. Utility of dysphagia screening results in predicting poststroke pneumonia. Stroke 2010;41:2849-2854. https://doi.org/10.1161/ STROKEAHA.110.597039

50 Di Pede C, Mantovani ME, Del Felice A, et al. Dysphagia in the elderly: focus on rehabilitation strategies. Aging Clin Exp Res 2016;28:607-617. 